\title{
Translation and validation of the Persian ICIQ bladder diary
}

\author{
Sona Tayebi ${ }^{1}$ (D) $\cdot$ Hanieh Salehi-Pourmehr ${ }^{2}$ (D) Sakineh Hajebrahimi ${ }^{1,2}$ (D) Hashim Hashim $^{3}$ (D)
}

Received: 5 March 2021 / Accepted: 25 May 2021 / Published online: 12 July 2021

(C) The International Urogynecological Association 2021

\begin{abstract}
Introduction and hypothesis The objective was to translate the International Consultation of Incontinence Questionnaire (ICIQ) bladder diary into Persian and validate it among the Iranian population with lower urinary tract symptoms.

Methods After obtaining permission from the ICIQ group to translate the ICIQ bladder diary into Persian, we translated, backtranslated it, and developed a Persian ICIQ bladder diary. Then, we evaluated its reliability and validity virtually via social media owing to some limitations caused by the COVID-19 pandemic. We calculated the content validity index (CVI) and content validity ratio (CVR) quantitatively.

Results Thirty-nine participants completed the final version of the ICIQ bladder diary. Most of the participants were female (76.3\%). For face validity, we interviewed 10 participants. Most of them considered it easy to complete the bladder diary, using a 500-ml measuring cup. Ten experts' panel determined the CVI, and the CVR. All ICIQ bladder diary criteria gained a high score, and all experts believed that all criteria of the diary were essential for patient evaluation (CVI: 0.89 to 1). Cronbach's alpha coefficient in all diary parameters was greater than 0.80 indicating high internal consistency. The test-retest reliability by intraclass correlation coefficients (ICC) for each diary parameters showed a high score indicating good agreement between the first and second test diaries.

Conclusion The Persian ICIQ bladder diary demonstrates good stability and strong content validity among the Iranian population with lower urinary tract symptoms.
\end{abstract}

Keywords International Consultation on Incontinence $\cdot$ Lower urinary tract symptoms $\cdot$ Bladder diary $\cdot$ Validation $\cdot$ Translation

\section{Introduction}

Lower urinary tract symptoms (LUTS) are among the most common complaints in urology clinics, with a profound impact on patient's quality of life [1]. LUTS are divided into storage (frequency, nocturia, urgency, etc.), voiding (intermittency, hesitancy, low stream urine, etc.), and post-micturition symptoms (post-void dribbling, etc.) [2]. In middle age, LUTS

Sakineh Hajebrahimi

hajebrahimis@gmail.com; ebrahimis@tbzmed.ac.ir

1 Urology Department, Faculty of Medicine, Tabriz University of Medical Sciences, Azadi Street, Golgasht Avenue, Tabriz, East Azarbaijan 5166/15731, Iran

2 Research Center for Evidence-based medicine, Iranian EBM Centre: A Joanna Briggs Institute (JBI) Center of Excellence, Tabriz University of Medical Sciences, Azadi Street, Golgasht Avenue, Tabriz, East Azarbaijan 5166/15731, Iran

3 Bristol Urological Institute, Southmead Hospital, North Bristol NHS Trust, Bristol, UK are more prevalent in women, whereas they are more common in men over the age of 70, despite urinary incontinence in women. Sixty percent of adults in Europe and North America and $61.2 \%$ of adult women older than 40 years in South-East Asia suffer from LUTS [3]. In a recent study in the central part of Iran, Zargham et al. showed that $95.5 \%$ of adult women referred to a urology clinic had at least one LUTS [4]. In addition to a detailed history taking and physical examination, self-reported questionnaires, and urinary diaries are beneficial in the initial management of LUTS, identifying the potential causes, and the extent of symptoms. Different types of self-reported questionnaires have been developed for this purpose, including the International Consultation of Incontinence Questionnaire-Lower Urinary Tract Symptoms for females and males (ICIQ- FLUTS and ICIQ-MLUTS) [5]. Although these questionnaires are beneficial in some respects, they fail to provide the full picture of symptoms owing to their subjective nature and the recall bias. More specifically, some patients may exaggerate their symptoms, whereas others may unknowingly underestimate their complications. One of the data collection methods that eliminates such subjectivities is 
the bladder diary, with objective documentation of bladder function in a real-time manner and recall bias reduction [6]. However, patient cooperation in completing the bladder diary plays an essential role in reducing recall bias.

According to the International Continence Society (ICS), three types of urinary diaries are available, including micturition time charts (only record the time of urination within a minimum duration of $24 \mathrm{~h}$ ), frequency-volume charts (considering the time and volume of urination in at least $24 \mathrm{~h}$ ), and bladder diaries (record the time and volume of urination, as well as other information such as the time, type, amount of fluid intake, urinary incontinence episodes, pad usage, and degree of urgency) [6].

Bladder diaries are part of the ICS urodynamics protocol and provide useful information for filling cystometry, such as maximum bladder capacity and functional bladder capacity.

Owing to the widespread use of bladder diaries in clinical practice and research, researchers are trying to design userfriendly electronic applications for recording the bladder diary, making the process easier for both patients and clinicians [7-9]. Considering the fact that $95.5 \%$ of adult Iranian women referred to urology clinic have at least one LUTS, the aim of the current study is to translate the ICIQ bladder diary and evaluate the validity and reliability of the Persian version of the ICIQ bladder diary, thus allowing the standardization of data collection for both clinical and research purposes.

\section{Materials and methods}

\section{Translation and back-translation process}

After obtaining permission from the ICIQ group to translate the ICIQ bladder diary into Persian, two Persian native speaker urologists translated the diary separately. Following review and comparison of these two versions, the final Persian version was developed, and then back-translated into English by two professional translators, who were not medical staff and were not familiar with the bladder diary. After the final review, the back-translated draft was sent to the ICIQ group for approval. Following agreement with them, the Persian version was checked by ten urologists for simplicity, comprehensibility, and accuracy of the content. All participating urologists had been exclusively working in the management of LUTS for more than 10 years. Owing to the appropriateness of the translated content, no change was made to the final Persian version.

\section{Validation of the Persian version of the ICIQ bladder diary}

Local ethical approval was obtained. First, an urologist interviewed virtually 10 participants who completed the 3day bladder diary, to assess their comments on the content, clarity, and simplicity of the bladder diary parameters. Data saturation was achieved, and data collection ended. Then we invited participants from across Iran to enroll in the study by virtual advertisement. Participants completed the questionnaire after the researcher explained the study objectives. Before data collection, we obtained written consent from all participants. The researcher emphasized the voluntary nature and confidentiality of the information.

\section{Sample size calculation and eligibility criteria}

Considering the main outcomes of study, the sample size was calculated as 74 cases, utilizing one proportion formula, and assumptions of $95 \%$ confidence, $80 \%$ power, and accepted difference of $60 \%$ in effect size. However, to increase the validity, 85 cases were considered.

Inclusion criteria were adults over 18 years of age, ability to read and write in Persian, and no cognitive disorders or acute urinary tract infections based on the absence of cystitis symptoms during the study period. We recorded participants' demographic characteristic and sent a Persian version of the bladder diary to each participant virtually. We asked all participants to complete a 3-day bladder diary with a 500-ml measuring cup, which was provided for all participants, with an interval of 3 to 7 days.

\section{Validity}

Content and face validity was assessed considering expert opinions. Both quantitative and qualitative methods were used to determine the content validity. To determine the content validity index (CVI), the research team asked 10 expert urologists in the field of LUTS management from all over Iran to complete a self-designed questionnaire based on the ICIQ bladder diary, using a four-point Likert scale (1 irrelevant, 2 slightly relevant, 3 relevant, 4 highly relevant), about the simplicity, exclusiveness, and clarity of the ICIQ bladder diary parameters. Item-CVI (I-CVI) was computed by the following formula: the number of experts giving a rating of "very relevant" for each item divided by the total number of experts. The amount of CVI in a range of 0 to 1 ; a score closer to 1 has a higher validity. Items with scores $>0.79$ were accepted, between 0.70 and 0.79 , the item needed revisions, and items with CVI $<0.7$ needed corrections.

The content validity ratio (CVR) was assessed by evaluating expert opinions on each bladder diary parameter, based on a three-part spectrum: "essential," "useful but not essential," and "not essential." The answers were finally calculated using statistical formulas of CVR $=(\mathrm{Ne}-\mathrm{N} / 2) /(\mathrm{N} / 2)$ in which the $\mathrm{Ne}$ is the number of panelists indicating "essential" and $\mathrm{N}$ is the total number of panelists. The calculated CVR numbers ranged from 0 to 1 ; this number was compared and confirmed considering its equivalent amount based on the number of 
experts using the "Lawshe" table [10]. The higher scores demonstrate significant agreement among panel experts [11].

To evaluate content validity qualitatively, we asked experts to provide feedback about bladder diary parameters according to the following criteria: correct grammar, appropriate word usage, and properly placed items.

\section{Internal consistency}

Internal consistency was assessed using the Cronbach alpha. Values between 0.70 and 0.90 were considered to be acceptable [10].

\section{Reliability}

To determine the bladder diary's reliability, we assessed the test-retest reliability results completed by participants with a 3- to 7-day interval.

\section{Statistical analysis}

First, all data were inputted into EXCEL (2013), and then analyzed using IBM SPSS 24.0 software. The data normality was evaluated using descriptive evidence. The quantitative and qualitative data used "mean \pm SD" and frequencies and percentages respectively, and Spearman analysis was used to calculate the correlation coefficient.

\section{Results}

Although, the sample size was calculated as 85 cases, the ICIQ bladder diary was delivered to 140 participants via social media owing to the limitations caused by the COVID-19 pandemic. However, only 48 were returned, due to the COVID19 pandemic and weak cooperation of participants in returning the diaries to the researcher; in addition, as some of the data were missing from the diaries, only 39 diaries had been completed correctly and were accepted into the study. Finally, considering the $p$ values in the study, and power analysis, in most of the participants, a power of more than $80 \%$ was achieved.

Most of the participants were female (76.3\%). The mean (SD) age was 42.0 (11.56) years (minimum 19, maximum 69). The results of the ICIQ bladder diary are shown in Table 1 .

\section{Validity}

For face validity, we interviewed 10 participants with different ages (range: 32-69 years old) and occupations (housewife, teacher, nurse, or physician) who had completed the ICIQ bladder diary. Most of them found the ICIQ bladder diary easy to complete, using a 500-ml measuring cup. However, some participants stated that the absence of an icon in the table of bladder diary for recording "wake up," and "sleep" time could lead to neglect of its documentation.

\section{Internal consistency}

We used Cronbach alpha to evaluate the internal consistency. Our results showed that the Cronbach alpha coefficient was 0.809 for drinking volume, 0.838 for voided volume, 0.870 for minimum voided volume, 0.846 for maximum voided volume, and 0.914 for urinary frequency. All of the diary parameters achieved a score greater than 0.8 , indicating good internal consistency.

\section{Reliability}

Thirty-nine subjects completed the ICIQ bladder diary twice with a 3- to 7-day interval. Parameters abstracted from the first and second ICIQ bladder diaries have a high correlation. The test-retest reliability was assessed by intra-class correlation coefficients (ICCs) for each parameter. The ICCs were high for all scales, indicating good agreement between the first and second test diaries (Table 2).

In our evaluation, I-CVI for all dimensions of the ICIQ bladder diary value was 1 and the majority of items were considered relevant. In terms of CVR, all ICIQ bladder diary criteria gained a high score, and all experts believed that all criteria of the diary were essential for patient evaluation (Table 3).

\section{Discussion}

The ICIQ bladder diary records urinary frequency, voided volume, and fluid intake (amount, time, and type) as well as bladder sensation and pad usage. A bladder diary is an accurate, affordable, quantitative, and inexpensive tool for evaluating LUTS and guiding behavioral modifications, which improves treatment results by actively involving the patient in the treatment process [6].

The current study translated and determined the validity and reliability of the Persian version of the ICIQ bladder diary among the Iranian population. LUTS are common conditions in the Iranian population, and at least $95 \%$ of adult females has had one of the LUTS [4]. Considering the bladder diary as an important tool for evaluating LUTS, determination of its validity and reliability are required. For this purpose, cultural adaptation of the diary has great importance. To use this diary in our clinical setting, after translation and back-translation, we considered the minor revisions comments of the ICIQ group, which were emailed to us. According to our results, the Persian version of ICIQ bladder diary has good face validity. To determine the content validity, we asked expert 
Table 1 The parameters of International Consultation of Incontinence Questionnaire (ICIQ)-bladder diary in the first and second tests

\begin{tabular}{|c|c|c|c|c|c|c|}
\hline \multirow[b]{2}{*}{ Diary parameter $(N=39)$} & \multicolumn{3}{|c|}{ First test diary } & \multicolumn{3}{|c|}{ Second test diary } \\
\hline & Minimum & Maximum & Mean $(\mathrm{SD})^{\mathrm{a}} /$ median $(\mathrm{IQR})^{\mathrm{b}}$ & Minimum & Maximum & Mean $(\mathrm{SD})^{\mathrm{a}} /$ median $(\mathrm{IQR})^{\mathrm{b}}$ \\
\hline Drinks per $24 \mathrm{~h}, \mathrm{ml}$ & 1,040 & 2,600 & $1,842(462.52)^{\mathrm{a}}$ & 1,270 & 2,400 & $1,766.67(396.01)^{\mathrm{a}}$ \\
\hline Total $24 \mathrm{~h}$ voided volume, $\mathrm{ml}$ & 690 & 2,700 & $1,373(707.16)^{\mathrm{a}}$ & 550 & 2,600 & $1,592.67(667.72)^{\mathrm{a}}$ \\
\hline Voided frequency per $24 \mathrm{~h}, n$ & 4 & 14 & $7.06(2.61)^{\mathrm{a}}$ & 4 & 14 & $7.00(2.40)^{\mathrm{a}}$ \\
\hline Maximum voided volume, $\mathrm{ml}$ & 120 & 720 & $342(128.70)^{\mathrm{a}}$ & 120 & 600 & $356.22(113.71)^{\mathrm{a}}$ \\
\hline Minimum voided volume, $\mathrm{ml}$ & 20 & 300 & $137(83.73)^{\mathrm{a}}$ & 30 & 350 & $154.59(83.49)^{\mathrm{a}}$ \\
\hline Bladder sensation score $0, n$ & 0 & 9 & $0(0,2)^{\mathrm{b}}$ & 0 & 9 & $0(0,2.5)^{\mathrm{b}}$ \\
\hline Bladder sensation score $1, n$ & 0 & 12 & $5(1,6)^{b}$ & 0 & 12 & $5(1,6)^{b}$ \\
\hline Bladder sensation score $2, n$ & 0 & 3 & $0(0,0)^{\mathrm{b}}$ & 0 & 3 & $0(0,0)^{\mathrm{b}}$ \\
\hline Bladder sensation score $3, n$ & 0 & 4 & $0(0,1)^{\mathrm{b}}$ & 0 & 4 & $0(0,1)^{\mathrm{b}}$ \\
\hline Bladder sensation score $4, n$ & 0 & 0 & $0(0,0)^{\mathrm{b}}$ & 0 & 0 & $0(0,0)^{\mathrm{b}}$ \\
\hline Nocturia, $n$ & 0 & 1 & $1.0(1.0)^{\mathrm{b}}$ & 0 & 2 & $1.0(0.0)^{\mathrm{b}}$ \\
\hline Leaks in $24 \mathrm{~h}, n$ & 0 & 0 & $0^{\mathrm{b}}$ & 0 & 0 & $0^{\mathrm{b}}$ \\
\hline
\end{tabular}

${ }^{a}$ In cases with normal distribution, mean (standard deviation) were reported

${ }^{\mathrm{b}}$ For nonparametric data, values are reported as median (interquartile range)

panels of urologists from different academic centers all over Iran to complete the related questions in terms of bladder diary assessment purpose, clarity, aid in LUTS diagnosis, and easy comprehension of the items. The results showed a high rate of content validity in terms of the purpose of bladder diary assessment.

Similar to our results, Shen et al. translated and validated ICIQ bladder diary among 146 Chinese women with LUTS. The experts' authority was $0.94 \pm 0.06$, and the I-CVI was 0.909-1.000. The scale CVI was 0.974, which showed that the Chinese ICIQ bladder diary had good stability, content validity, and rigorous responsiveness among Chinese women with LUTS [5].

Although bladder sensation was simple and easy to understand for patients in this diary, some participants did not answer these questions, and it seems that it would be better for

Table 2 Comparison of test-retest diary parameters $(n=39)$

\begin{tabular}{llll}
\hline Diary parameters $(N=39)$ & r* & $n$ & ICC (95\% CI $)$ \\
\hline Drinks per 24 h, ml & 0.714 & $<0.001$ & $0.857(0.470-0.962)$ \\
Total 24 h voided volume, $\mathrm{ml}$ & 0.654 & $<0.001$ & $0.886(0.837-0.897)$ \\
Maximum voided volume, $\mathrm{ml}$ & 0.717 & $<0.001$ & $0.843(0.694-0.919)$ \\
Minimum voided volume, $\mathrm{ml}$ & 0.708 & $<0.001$ & $0.870(0.7-7-0.933)$ \\
Voided frequency per 24 h, $n$ & 0.785 & $<0.001$ & $0.912(0.821-0.957)$ \\
Bladder sensation score $0, n$ & 0.856 & $<0.001$ & $0.866(0.796-0.920)$ \\
Bladder sensation score $1, n$ & 0.992 & $<0.001$ & \\
Bladder sensation score 2, $n$ & 0.895 & $<0.001$ & \\
Bladder sensation score 3, $n$ & 0.964 & $<0.001$ & \\
Bladder sensation score 4, $n$ & - & - & \\
\hline
\end{tabular}

*Spearman correlation these items to be highlighted more, and that caregivers who deliver the diary to patients emphasize the completion of them. In terms of test-retest reliability of the ICIQ bladder diary, our results showed the stability of this diary. Our results revealed a strong correlation between the first and second diary parameters using Spearman's test, similar to the Chinese version [5].

The ICIQ bladder diary can be used in both a clinical and a research setting. According to the guidelines, a 3-day bladder diary is the shortest form of a diary to guarantee the diary parameters. Bright et al. developed the first validated bladder diary, which has been used as a common instrument for assessing LUTS worldwide [6, 12]. Bright et al. determined the construct validity of the urinary diaries in 264 patients. The patients completed ICIQ-LUTS questionnaires too, and the researchers observed a good agreement between questionnaire and diary recordings of nocturia $(\kappa=0.653 ; p<0.001$; $92.2 \%)$; incontinence $(\kappa=0.351 ; p<0.001 ; 64.5 \%)$, and urodynamic findings ( $\mathrm{K}=0.378 ; p<0.001 ; 69.2 \% ; n=104)$. However, in urgency, there was a weak agreement with questionnaire responses $(\kappa=-0.215 ; p<0.001 ; 36 \%)$ and urodynamic observations $(\mathrm{K}=-0.105 ; p=0.256 ; 43.7 \%)$ [12].

One of the strong points of our study was the good and acceptable internal consistency using Cronbach alpha in all of the diary parameters. In addition, test-retest for reliability assessment using ICCs showed a good agreement between the first and second test diaries, indicating that the ICIQ bladder diary has good internal consistency in addition to acceptable reliability.

We delivered the ICIQ bladder diary for 140 participants via social media owing to the limitations caused by the 
Table 3 Evaluation the item content validity index $(I-C V I)$, and content validity ratio $(C V R)$ of the International Consultation of Incontinence Questionnaire bladder diary $(I C I Q-B D)$ by ten experts

\begin{tabular}{|c|c|c|c|c|}
\hline Items & $\begin{array}{l}\text { Necessity } \\
(\text { CVR) }\end{array}$ & $\begin{array}{l}\text { Simplicity } \\
(\mathrm{CVI})\end{array}$ & $\begin{array}{l}\text { Transparency } \\
(\mathrm{CVI})\end{array}$ & $\begin{array}{l}\text { Relevancy } \\
\text { (CVI) }\end{array}$ \\
\hline ICIQ-BD has clear assessment purposes & 1 & 1 & 1 & 1 \\
\hline ICIQ-BD has a clear form & 0.78 & 1 & 1 & 1 \\
\hline ICIQ-BD's content is easy to understand & 1 & 1 & 0.89 & 1 \\
\hline ICIQ-BD can help diagnose & 1 & 1 & 1 & 1 \\
\hline $\begin{array}{l}\text { ICIQ-BD is recommended as a basic assessment tool for lower urinary tract } \\
\text { symptoms }\end{array}$ & 0.78 & 1 & 1 & 1 \\
\hline
\end{tabular}

COVID-19 pandemic. However, only 48 returned them, and only 39 diaries were acceptable and had been completed correctly. Owing to missing data, including the amount of voided volume, or fluid intake volume, as well as bladder sensation, 9 diaries out of 48 were excluded from the study. Although, some participants $(n=11)$ were health-care providers in our study, they were not clinicians or nurse practitioners, and their main activity in health centers was only as an administrator or staff member. Therefore, it may have a minimal effect on the results of our study. However, similar to the previous study on Chinese women, our study demonstrated that the ICIQ bladder diary could be a credible and effective assessment tool in a clinical setting and in scientific research on the Iranian population.

\section{Conclusion}

The Persian version of the ICIQ bladder diary is a valid and reliable tool with strong content validity among the Iranian population with LUTS, allowing standardized assessment of patients in Iran. This would also allow Iranian patients to be included in clinical trials utilizing bladder diaries as well as comparison of patient groups across the world.

Acknowledgements We would like to thank Research and the vicechancellor of Tabriz University of Medical Sciences for financial support and ethical approval (Grant NO: 66737).

Contributions Sona Tayebi, Sakineh Hajebrahimi, Hanieh SalehiPourmehr: project development, data collection; Hanieh SalehiPourmehr, Sona Tayebi: manuscript writing, data analysis; Sakineh Hajebrahimi, Hashim Hashim: manuscript editing. All authors read and approved the final version of the manuscript.

\section{Declarations}

Conflicts of interest The authors have no conflicts of interest to declare that are relevant to the content of this article.

\section{References}

1. Girman CJ, Jacobsen SJ, Tsukamoto T, Richard F, Garraway WM, Sagnier PP, et al. Health-related quality of life associated with lower urinary tract symptoms in four countries. Urology. 1998;51(3): 428-36. https://doi.org/10.1016/s0090-4295(97)00717-6.

2. Haylen BT, de Ridder D, Freeman RM, et al. An International Urogynecological Association (IUGA)/International Continence Society (ICS) joint report on the terminology for female pelvic floor dysfunction. Int Urogynecol J. 2010;21(1):5-26. https://doi.org/10. 1007/s00192-009-0976-9.

3. Chapple C, Castro-Diaz D, Chuang Y-C, Lee K-S, Liao L, Liu S-P, et al. Prevalence of lower urinary tract symptoms in China, Taiwan, and South Korea: results from a cross-sectional, population-based study. Adv Ther. 2017;34(8):1953-65.

4. Zargham M, Pourmomeny AA, Soltanmohamadi M. The prevalence of lower urinary tract symptoms (LUTS) and incontinence in Iranian women. Urol J. 2020;17(3):276-80.

5. Shen L, Hou L, Li B, Jin X, Han F, Wang Y. Translation of the ICIQ-bladder diary and its validation among Chinese females with lower urinary tract symptoms. Int Urogynecol J. 2020;31(12): 2535-42. https://doi.org/10.1007/s00192-020-04339-9.

6. Bright E, Drake MJ, Abrams P. Urinary diaries: evidence for the development and validation of diary content, format, and duration. Neurourol Urodyn. 2011;30(3):348-52.

7. Chun K, Kim SJ, Cho ST. Noninvasive medical tools for evaluating voiding pattern in real life. Int Neurourol J. 2017;21(Suppl 1):S10.

8. Mateu Arrom L, Peri Cusi L, Franco de Castro A, López-Fando L, Jiménez Cidre MÁ, Alcaraz Asensio A. Development and feasibility assessment of a 3 day electronic bladder diary as an app for smart-phone. Neurourol Urodyn. 2018;37(5):1717-23.

9. Cameron AP, Wiseman JB, Smith AR, Merion RM, Gillespie BW, Bradley CS, et al. Are three-day voiding diaries feasible and reliable? Results from the symptoms of lower urinary tract dysfunction research network (LURN) cohort. Neurourol Urodyn. 2019;38(8): 2185-93.

10. Lawshe $\mathrm{CH}$. A quantitative approach to content validity. Pers Psychol. 1975;28(4):563-75.

11. Valim MD, Marziale MHP, Hayashida M, Rocha FLR, Santos JLF. Validity and reliability of the questionnaire for compliance with standard precaution. Rev Saude Publica. 2015;49:87.

12. Bright E, Cotterill N, Drake M, Abrams P. Developing and validating the International Consultation on Incontinence questionnaire bladder diary. Eur Urol. 2014;66(2):294-300.

Publisher's note Springer Nature remains neutral with regard to jurisdictional claims in published maps and institutional affiliations. 